\title{
Non-Orthogonal Transmission Techniques for Multibeam Satellite Systems
}

\author{
Ana I. Perez-Neira, Marius Caus and Miguel Angel Vazquez
}

\begin{abstract}
Non-orthogonal transmission is a promising technology enabler to meet the requirements of the forthcoming 5G communication systems. Seminal papers demonstrated that non-orthogonal multiplexing techniques outperform orthogonal schemes in terms of capacity, latency and user fairness. Since it is envisioned that satellites will be an integral component of the $5 \mathrm{G}$ infrastructure, it is worth studying how satellite communication systems can benefit from the application of non-orthogonal transmission schemes as well. Contrary to common perception, communications through a satellite present a different architecture and face different impairments than those in the wireless terrestrial links. This highlights that a general overview is needed to gain insight into the satellite payload architecture. In particular, this works aims at describing different non-orthogonal schemes and payload architectures that are suitable for the satellite environment. In this regard, a novel taxonomy is presented based on different multibeam transmission schemes. Finally, guidelines that open new avenues for research in this topic are provided.
\end{abstract}

\section{INTRODUCTION}

Non-orthogonal transmission schemes, such as powerdomain non-orthogonal multiple access (NOMA), may play a pivotal role in $5 \mathrm{G}$ to help increasing the peak data rate, the area traffic capacityand the spectral efficiency [1], [2]. From the theory, it is known that in certain communication channels, NOMA outperforms orthogonal schemes, such as frequency or time division multiplexing. This happens in particular in those scenarios where there is a large imbalance among user link qualities. At the receiver side, multiuser detection (MUD) techniques, such as linear detection and non-linear detection based on parallel or successive interference cancellation (SIC), can be utilized to decode the superimposed signals.

In parallel to the $5 \mathrm{G}$ evolution, the ever growing demand for high data throughput has triggered research efforts to improve the spectral efficiency of satellite communications. Very high or high throughput satellites (V/HTS) [3] are emerging satellite solutions that highly demand such spectral efficiency improvements. These systems deploy multiple beams that tessellate the coverage area in small beam footprints; thus, allowing frequency reuse across the coverage area. By adopting three or four frequency reuse schemes among beams it is practically guaranteed certain level of isolation

A. I. Perez-Neira is with the Centre Tecnològic de Telecomunicacions de Catalunya and the Universitat Politècnica de Catalunya.

M.Caus and M. A. Vazquez are with the Centre Tecnològic de Telecomunicacions de Catalunya. between co-channel beams. In this way, the interference, which comes from neighboring beams operating at the same frequency, is significantly lower than the desired signal. Therefore, the user terminals can apply single user decoding (SUD), which entails treating inter-beam interference as noise, without significant performance degradation. With the aim of lowering the cost per bit/s and increasing the spectral efficiency or the available system bandwidth, new systems foster more aggressive frequency reuse and thus, the inter-beam interference increases. To tackle this increase of the interference levels, existing satellite standards, such as the digital video broadcasting over satellite (DVB-S2X), have included provisions for implement multibeam precoding techniques in the forward link, which use the knowledge of the users' channels at the transmitter in order to precompensate for the interference.

It is envisioned that the role of satellites will be crucial in the forthcoming $5 \mathrm{G}$ networks to increase the availability in underserved areas. Hence, analogously to terrestrial communications, it is deemed necessary to study if implementations of non-orthogonal transmission techniques can further increase the spectral efficiency of satellite communications. In this article we aim at benefiting from the penetration of non-orthogonal schemes in $5 \mathrm{G}$ to study satellite schemes with shared time-frequency resources. However, the work presented is not limited to conventional NOMA but explores different non-orthogonal transmission techniques suitable for the downlink of multibeam satellite systems. Concerning the practical aspects, it is worth emphasizing that the satellite payload is equipped with a multiplicity of feeds, which allow simultaneously serving different spot beam areas on the ground. Typical multibeam architectures do not support power sharing, which implies that each feed has its own power budget. Therefore, the peculiarities of multibeam satellite communications call for specific transmission strategies, precoding techniques and ultimately, new network resource allocation strategies. It is worth mentioning that there are papers that review precoding techniques in multibeam satellites [4]. On the contrary, there is not already an appropriate tutoriallyoriented article that encompasses non-orthogonal transmission techniques beyond precoding and NOMA that account for the unique characteristics of satellite communications. With this aim, this article introduces an overview taxonomy based on different multibeam transmission schemes. 


\section{领}

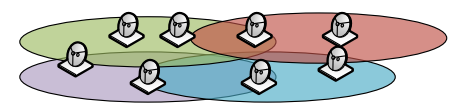

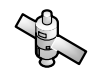

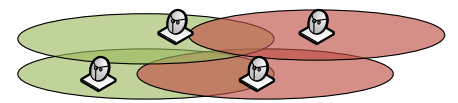

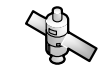

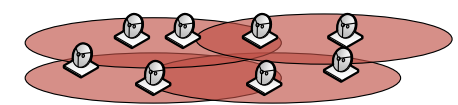

Fig. 1: Different interfering satellite architectures in the case of four isolated beams with at most two simultaneous users per beam: a) 4-colour frequency reuse; b) 2-colour frequency reuse; c) Full frequency reuse.

The rest of the article is organized as follows: Section II presents the basic idea behind NOMA and lays out the different non-orthogonal transmission schemes that arise in the satellite multibeam architecture. Next, Section III explains how non-orthogonal transmission schemes can be integrated with precoding in order to notably improve spectral efficiency in a realistic environment with full frequency reuse, whenever there is full channel state information at transmission (CSIT). Finally, Section IV draws some conclusions and outlines further research directions.

\section{REFERENCE SCHEMES OF NON-ORTHOGONAL TRANSMISSION TECHNIQUES IN V/HTS}

To briefly introduce NOMA, we focus the attention on its simplest form that serves two users by employing power-domain multiplexing [2]. One of the users receives a markedly stronger signal than the other. The other user is referred to as the weak user, which can be, for instance, closer to the edge of the beam, or equipped with a smaller receiving antenna. In NOMA, this asymmetry is usually harnessed by assigning more transmit power to the weak user. As for the decoding strategy, the weak user recovers the corresponding message by treating the interference as noise with SUD, while the strong user can recover the desired message by resorting to MUD strategies. That is, the user receives the interference strong enough so that it can be decoded first, and then performs SIC to recover the desired message.

Bearing in mind the potential of NOMA, this article describes different forms of non-orthogonal transmission that can be applied in diverse satellite multibeam schemes or architectures, which are sketched in Figure 1. That is, we extend the current notion of NOMA in order to incorporate additional transmission and receiver operations which are beneficial for the multibeam satellite system scenario. The multibeam nature of the satellite opens a range of possible non-orthogonal transmission schemes, which, to the authors' knowledge, is the first time that they are systematically studied within the satellite context. For the sake of clarity in the exposition, Figure 1 isolates just four beams among the possible $K$ beams in a V/HTS geostationary orbit (GEO) satellite.
The satellite has a transparent payload, i.e., all the processing is done at the gateway. In this section, we will discuss the design issues in unicast transmission for nonaggressive and aggressive frequency reuse schemes.

\section{A. Non-aggressive frequency reuse among beams}

First, we refer to Figure 1a, which draws a perfect analogy between one satellite beam and one terrestrial cell. In this case, satellite communications can directly benefit from the theory developed in the wireless terrestrial NOMA framework. Figure 1a represents the situation when the frequency reuse among beams is not very aggressive (e.g. by adopting three or four frequencies), which paves the way to applying NOMA on a per beam basis. Note that, within a single beam, NOMA can be envisaged as long as there is a significant signal-to-noise ratio (SNR) imbalance among users [3]. In GEO satellite the near-far problem of terrestrial network does not exist. In a terrestrial network the difference in received power could reach 20-30 dB. Such variation typically does not exist in GEO satellite. However, there are two cases that is worth exploring: i) very low Earth orbit where the difference in path and antenna gain could be very large and ii) non-homogeneous user equipment. In GEO satellite communications, the SNR imbalance appears when distinct classes of user terminal are deployed. Namely, user terminals with different receiving antenna gains, which includes the scenario of fixed satellite services in coexistence with aeronautical or vehicular users that are connected via satellite [5], [6].

For what concerns the satellite air-interface, it is important to remark that the bandwidth is usually split into a moderate number of carriers. Therefore, the transmitted signal exhibits low or mild envelope fluctuation, which is desirable to mitigate the nonlinear effects induced by the satellite transponder and guarantee a high-power amplifier efficiency. Clearly, the immediate consequence of applying NOMA is that sudden peaks or drops may happen more frequently, since signals are sent via additive superposition. This highlights that pre-distortion is necessary to reduce the power back-off and make the most efficient use of the power and the spectrum resources in the satellite. The advent of onboard processors paves the way to using adaptive pre- 


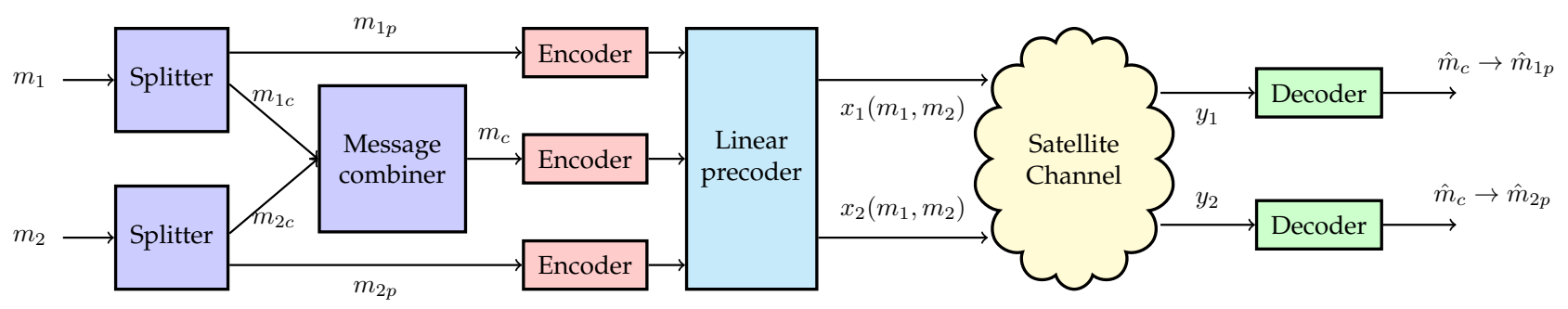

Fig. 2: Encoder and decoder structure for the two-user MISO-BC with rate splitting.

distortion, which is a very promising solution to relay non-orthogonal signals through the satellite payload. The same conclusion is valid for the different forms of non-orthogonal transmission that will be addressed in the following.

\section{B. 2-colour frequency reuse schemes}

As we have pointed out, to unleash the full potential of multibeam satellite communication systems, it is deemed necessary to adopt more aggressive frequency reuse schemes. For the sake of clarity, and to better illustrate the concept of non-orthogonal transmission in V/HTS, we consider a beam pattern with a reuse factor 2. To simplify the scenario, a clustering algorithm can be executed to pair the beams according to the location of the users to be served. If the pairing is made to minimize the interference from outside the beam pair at the location of users, then a high level of abstraction can be made by regarding the global multibeam system as a set of multiple two-beam and two-user communication systems. In each beam pair the frequency is reused to serve two users, as illustrated in Figure $1 \mathrm{~b}$. The signals that come from outer beams contribute to the background interference, which is treated as noise. In this case, non-orthogonal techniques shall be designed to cope with the interference generated by inner beams.

If there is no beam cooperation, we can draw an analogy with the two-user interference channel (IC), which contrasts with the broadcast channel (BC) that usually comes up in the cellular architecture. In the IC, characterized by the Han-Kobayashi (HK) capacity inner bound, close-to-optimal strategies consist in dividing each user's message into common and public messages, which are sent via superposition coding. This opens the door to the so-called rate splitting approaches [7], whose implementation has to take into account that the common message is decodable by both users, while the private one has to be recovered only by its intended receiver and it is not decodable by the other one.

If beams are allowed to cooperate in the scenario represented in Figure 1b, then the multiple-input singleoutput BC (MISO-BC) model becomes now the reference. The centralized control that the satellite gateway has on the beams naturally favours the application of precoding techniques [4]. Recently, some works have examined the practicality of combining precoding and rate splitting. The idea is illustrated in Figure 2. At the transmit side, each user's message is split into two parts. Without loss of generality, if $m_{i}$ denotes the message intended to the $i$ th user, then $m_{i c}$ and $m_{i p}$ correspond to the common and the private messages, respectively. Unlike the HK superposition signaling, common parts can be combined into one. The resulting messages are independently encoded and linearly precoded. At the receive side, it is common practice to decode first $m_{c}$ and then apply SIC to decode the corresponding private message. To alleviate the complexity, a two-step approach has been proposed in [8]. The rationale is to conceive a low-complexity solution, where the design of the precoders associated with the private and the common messages is decoupled. At the expense of degrading the performance, the complexity can be further reduced and the feedback requirements can be relaxed just by exploiting partial CSIT in the form of SNR [9]. It is important to remark that most of the works addressing the transmit beamforming with common information decoding are usually subject to a global power constraint, e.g. [7], rather than the perfeed constraints that have to be imposed in the satellite payload.

Remarkably, if the beams cooperate and rate splitting is employed, channel imbalance between the two users results not to be so imperative. As an example, Figure 3 illustrates that rate splitting achieves a larger rate region than that of orthogonal schemes. The variant with full and partial CSIT corresponds to the practical solution devised in [8] and [9], respectively. In this example, it has been considered that the SNR and interference-tonoise ratio (INR) are the same for each user at each beam. In satellite communications the multipath effect is usually weak while the line-of-sight component is very strong. For this reason, the envelope of the channel in the simulations has been modeled as a nonrandom constant and thus, the impact of the fading has not been evaluated. Figure 3a represents the situation where users are close to the center of the beam and as a consequence, the interference is weak. Interestingly, rate splitting outperforms frequency division multiplexing (FDM) by only exploiting partial CSIT. If full CSIT is available, rate splitting is able to provide higher rates than the zero-forcing ( $\mathrm{ZF}$ ) precoding. As users move to the overlapping area of two beams, the SNR decreases 


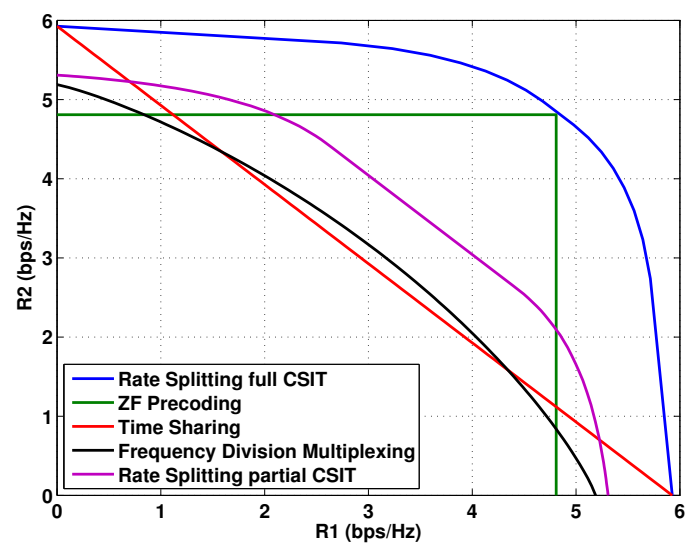

(a) $\mathrm{SNR}_{1}=\mathrm{SNR}_{2}=15.5 \mathrm{~dB}, \mathrm{INR}_{1}=\mathrm{INR}_{2}=5 \mathrm{~dB}$

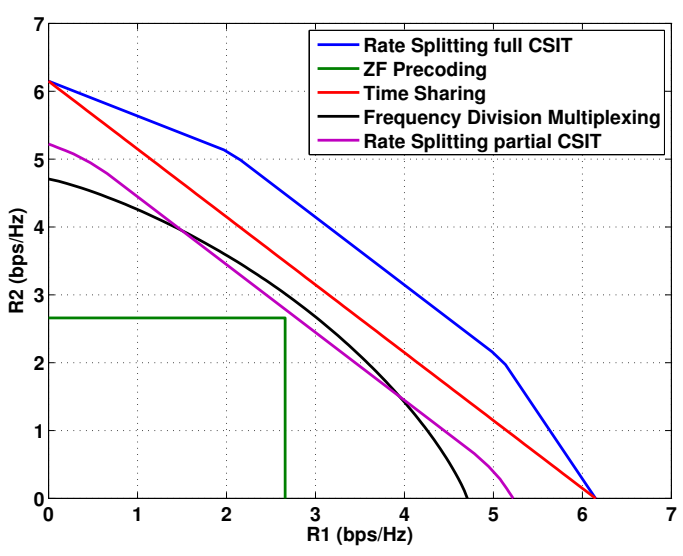

(b) $\mathrm{SNR}_{1}=\mathrm{SNR}_{2}=14 \mathrm{~dB}, \mathrm{INR}_{1}=\mathrm{INR}_{2}=10.5 \mathrm{~dB}$

Fig. 3: Achievable rate regions for different strategies that are applied to a two-beam and two-user communication system.

and the INR increases. This scenario is analyzed in Figure $3 b$. Numerical results reveal that ZF precoding is not able to remove the interference and preserve a high SNR. Furthermore, when the desired and the interfering signals are received with similar magnitude, rate splitting with partial CSIT may not be advantageous over FDM. In the light of these results it can be concluded that in the interference limited regime, it is crucial to leverage on rate splitting and full CSIT.

To demonstrate that the SNR imbalance between beam-center and beam-edge users is moderate, we have represented in Figure 4 the SNR that static users confined in the area of two adjacent beams would experience in clear sky conditions. It is worth mentioning that the dominant source of co-channel interference is limited to the adjacent beam. Using the satellite antenna radiation pattern and the system parameters provided by the European Space Agency (ESA), we have computed the SNR in approximately 360 positions for each beam. To establish a connection between the numerical results and the footprint, we have indicated the positions that would yield the SNRs and the INRs that have been considered in Figure 3. In other words, Figure $3 a$ and Figure $3 b$ represent the rates that are jointly achievable by the two users that form group 1 and 2, respectively.

Building upon the canonical models shown in Figure 1 , the multibeam nature can be used jointly with nonorthogonal transmission in order to increase the spectral efficiency. It must be mentioned that in satellite communications, the adoption of full frequency reuse schemes combined with precoding techniques is the leading paradigm to increase the spectral efficiency. A simplified scenario with four beams and two users per beam is represented in Figure 1c. The performance when all beams operate on the same frequency can be further improved by reaping the benefits of advanced decoding strategies, such as simultaneous non-unique decoding (SND). To show it, next section extends the analysis in

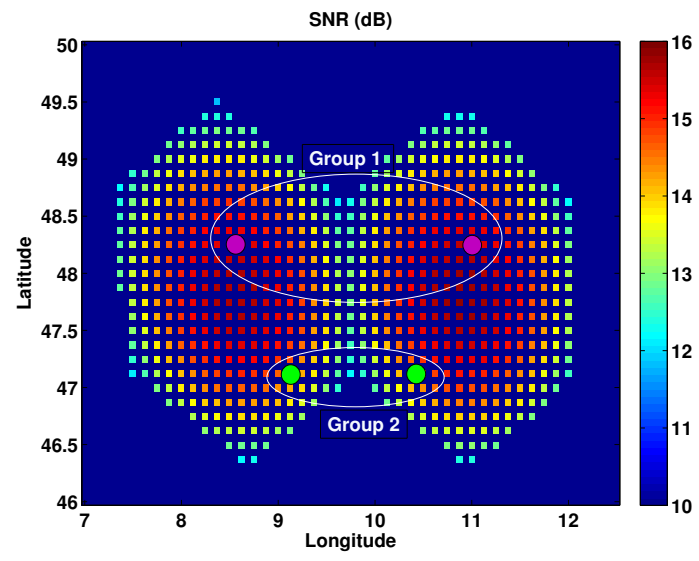

Fig. 4: Received SNR in two adjacent beams.

a more realistic environment, where one satellite has control over a large number of beams that operate on the same frequency.

\section{NON-ORTHOGONAL TRANSMISSION IN FULL FREQUENCY REUSE MULTIBEAM SATELLITES}

Recent advances in multibeam satellite systems have identified spatial precoding as a key enabler for increasing the spatial multiplexing gain [4]. If the V/HTS controls $K$ beams, these are created with $N(N \geq K)$ feeds or antenna elements onboard. Spatial precoding allows the transmission over a full frequency reuse system (i.e. all beams share the same spectrum resources) by jointly processing at the gateway the input signal in order to spatially mitigate the co-channel interference. In this context, the user terminals can benefit from a large available bandwidth preserving a sufficiently large signal-to-interference-plus-noise ratio (SINR) value. It is worth mentioning that user terminals can employ SUD at reception because the interference has been precanceled at the transmit side. 
In this section we incorporate the possibility of transmitting $L$ non-orthogonal frames within one beam in a system with full frequency reuse among beams, yielding a multi-layer transmission. In the proposed scenario, the number of transmission layers per beam, the number of beams and the number of feeds are related as follows: $N<K L$. This configuration reveals that there are not enough spatial degrees of freedom to eliminate the cochannel interference. As a matter of fact, having a lower number of feed elements than the number of frames being transmitted yields to both large intra-beam and interbeam interference (i.e. interference between users located at the same beam and different beams, respectively). This communication setting has also been reported in [10] for the NOMA cellular scenario. In terrestrial communications, the clusters in each cell are composed by one cellcenter user and one cell-edge user. Optimizing the precoding matrix of a downlink NOMA transmission with multiple clusters requires computationally demanding methods as reported in [11]. Due to that, alternative precoding designs based on closed-form methods have appeared to tackle the cellular scenario. In this context, a suboptimal approach to mitigate inter-cell interference is based on sharing the beamformer among the users that are located in the same cell [10]. To guarantee the success of this option, user clustering has to be devised so that selected users have a strong channel correlation. Upon mitigating the leakage between cells, NOMA comes naturally to tackle intra-cell interference, since users within each cell are judiciously selected to exhibit a high SNR imbalance. This strategy is usually referred to as multicluster multiple-input multiple-output NOMA (MIMONOMA). In the following, the novel aspects studied in the multibeam satellite domain are introduced.

In satellite communications, mimicking the strategy adopted in terrestrial communications may not always be valid to deal with inter-beam interference and increase the beam-edge users' throughput. As it has been previously explained, SNR imbalance is not dependent on the geographic position of users, but on the class of the receiver terminals. Hence, if beam-center and beamedge users employ the same class of terminals, the user channel conditions are similar and thus, the key feature of NOMA cannot be exploited. The detrimental situation that is created due to the peculiarity of the satellite channel has motivated a new approach presented in [12], [13]. Contrary to multi-cluster MIMO-NOMA, the idea is to refrain from sharing beamformers. Interestingly, the authors in [12] describe a precoding matrix design considering a scenario where the number of served users is twice the number of antenna elements. It has been shown that solutions based on low-complexity mechanisms like minimum mean square error precoding succeed in reducing inter-beam interference, by pairing users with highly correlated channels. The communication system that results from the scheme envisaged in [12], [13], which is called joint precoding and MUD (JPMUD), allows representing each beam with two sender-receiver

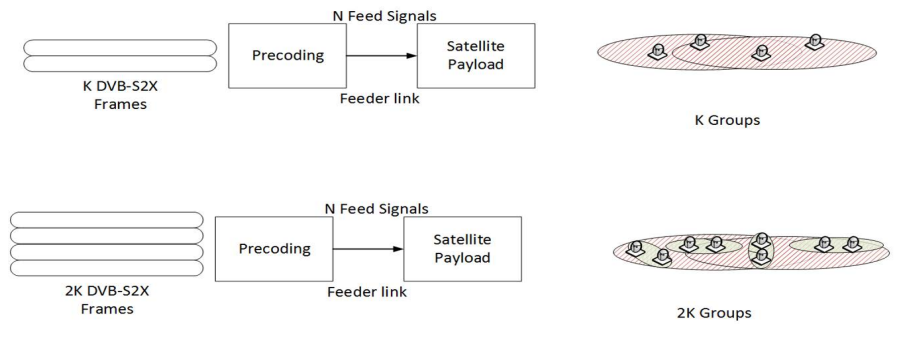

Fig. 5: Up: Precoding with single-layer transmission. Down: Precoding with multi-layer transmission.

pairs. This motivates the deployment of advanced receivers able to handle the remaining intra-beam interference. The main rationale of JPMUD techniques is that whenever the gateway aims at transmitting a number of frames $F=K L$ superior to the number of feed elements $N$, receivers with MUD capabilities can increase the overall data rates. Different MUD techniques are investigated and namely, the receiving strategy that leads to the highest system achievable rates is inspired by SND [14].

SND has been proposed in diverse scenarios where signals conveying messages intended to different destinations appear superimposed at any given receiver. Similarly to SND, the idea of the scheme proposed in [12] is to examine a subset of messages that is not limited to the message of interest. Then, depending on the SNR and the INR regimes of operation, the receiver either performs MUD or SUD. If the conditions are such that the interfering messages can be perfectly decoded, then the receiver ends up performing MUD, but unwanted messages are discarded after detection. In contrast, if the receiver fails to decode messages meant to other destinations, then SUD is performed. This strategy is advantageous for a number of reasons, notably the capacity of exploiting the structure of unwanted messages or treating them as noise as appropriate. This advanced receiver is proposed in [12] in contrast to current cellular approaches that rely on a pre-defined strategy, which usually encompasses SIC and SUD. The results show that despite there is not a clear channel gain imbalance between users served in the same beam, JPMUD under SND framework offers a remarkable sum-rate gain with respect to the case where precoding transmission tackles a number of frames $F=N$.

A second differentiating element in the application of JPMUD is the scheduling. It is known that user scheduling can enhance the attainable rates of spatial precoding. When JPMUD is addressed, the scheduling problem consists in pairing the user terminals in the most convenient way. In this context, the authors in [13] present different scheduling alternatives for JPMUD systems under the same precoding and MUD receiver operation design guidelines reported in [12]. Based on the numerical results presented in [13], pairing users 


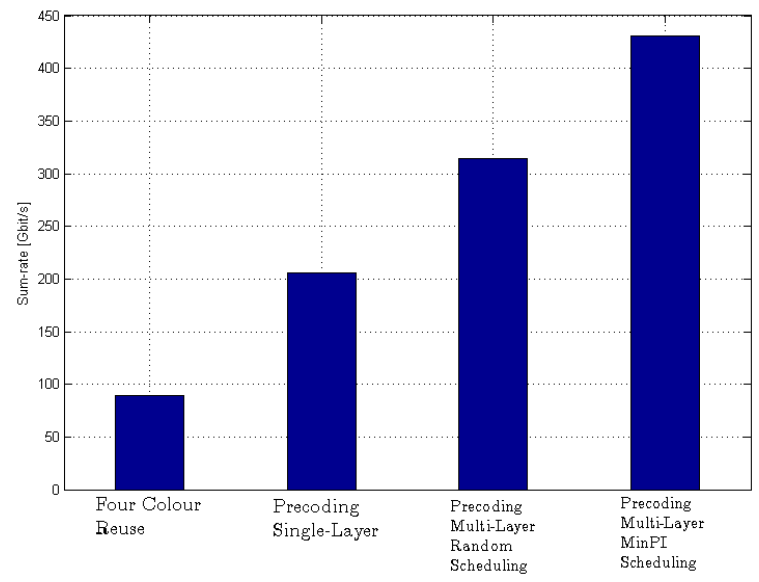

Fig. 6: Sum rate evaluation for multibeam transmission with different frequency reuse schemes and scheduling strategies.

with the lowest channel gain imbalance and Euclidean distance leads to the largest sum-rate values. Remarkably, this approach might not be convenient in other scenarios where SND is not the reference such as the ones described in [10].

Figure 5 depicts the resulting precoding satellite architecture for single- and multi-layer transmission. We note that, although the satellite payload can remain the same regardless of the transmission scheme, the feeder link, which goes from the gateway to the satellite, needs to increase its capacity in order to attend the new system requirements. Indeed, while conventional precoding multibeam satellite systems based on singlelayer transmission need a total feeder link bandwidth of $B K \mathrm{~Hz}$ (with $B$ as the user bandwidth), the application of JPMUD requires $2 B K \mathrm{~Hz}$.

In order to show the potential of the proposed transmission strategies in a full frequency reuse scenario, we perform a numerical evaluation over a close-toreal satellite scenario. Figure 6 depicts a system level evaluation of a multibeam satellite system with $N=245$ feed elements serving $K=245$ beams and a total number of $2 K=490$ users. We assume a maximum power per feed of 55 Watts and a power amplifier output back-off of $5 \mathrm{~dB}$. The channel model is the one reported in [4], where the antenna pattern has been shared by ESA.

Two benchmark cases have been included: the four frequency scheme without precoding and the precoding with single-layer transmission. Note that in both cases it has been considered a two time slot transmission in order to serve the 490 users with the considered 245 feed elements. The numerical results show that even with any type of scheduling, multi-layer transmission based on JPMUD presents a substantial sum rate gain compared to the benchmark case. In case the mentioned minimum power imbalance (MinPI) scheduling is used, a 109 and 384 percent gain is obtained with respect to the precoding for single-layer transmission case and the four frequency scheme.

\section{OPEN RESEARCH CHALLENGES AND CONCLUSIONS}

This article has reviewed the state-of-the-art in nonorthogonal transmission schemes for satellite mulitbeam communications and has provided a taxonomy of the main representative scenarios with respect to the order of the frequency reuse. Remarkably, it is still under research which is the most convenient way to distribute the network resources (i.e. power, beam and bandwidth), in order to take advantage of the potential flexibility of V/HTS and increase the access network capacity with non-orthogonal transmission techniques. We have noted that the framing of the multiuser data that is used in the satellite protocols is different from the one used in the terrestrial wireless standards. This is because current satellite communication systems need larger channel coding gains than terrestrial counterparts; each simultaneous frame transmission generally embeds more than one user terminal information, leading to the socalled multigroup multicast multibeam operation. This different framing has important consequences in the user scheduling and rate allocation. Therefore, these aspects motivate the need for different scheduling techniques as the one in [15], where the authors move from the work performed in [8] and propose a geographical scheduling algorithm in order to improve the fairness of a multicast precoder.

This article has shed some light on the role of nonorthogonal transmission in these flexible satellite systems; however, many aspects are yet to be defined and studied. There are pure physical layer aspects as: the impact of channel estimation errors at the receiver, the effect of timing offset between the signals transmitted by the different beams, low-complexity non-orthogonal coding/precoding and decoding strategies that leverage limited feedback or, a dynamic user scheduling and grouping strategies, to mention a few. These studies have to be cast with upper layers aspects, such as: nonuniform traffic distribution among beams, packet encapsulation and adaptive coding and modulation in nonorthogonal transmission, centralized or decentralized resource allocation, among others. The major advances have been carried out for centralized schemes. However, concerning decentralized ones, promising recent developments are within game theory as it was reported in [1] for the wireless cellular communications.

\section{ACKNOWLEDGMENTS}

The authors thank SatnexIV Network of Excellence, from the European Space Agency for the fruitful discussions. This work has received funding from the Spanish Government under grant TEC2017-88373-R (TERESA) and from the Catalan Government (2017-SGR-1479) Opinions, interpretations, recommendations and conclusions expressed herein are those of the authors and are not necessarily endorsed by the European Space Agency. 


\section{REFERENCES}

[1] L. Song, Y. Li, Z. Ding, and H. V. Poor, "Resource Management in Non-Orthogonal Multiple Access Networks for 5G and Beyond," IEEE Network, vol. 31, no. 4, pp. 8-14, July 2017.

[2] S. M. R. Islam, N. Avazov, O. A. Dobre, and K. Kwak, "PowerDomain Non-Orthogonal Multiple Access (NOMA) in 5G Systems: Potentials and Challenges," IEEE Communications Surveys Tutorials, vol. 19, no. 2, pp. 721-742, Secondquarter 2017.

[3] H. Fenech, A. Tomatis, S. Amos, V. Soumpholphakdy, and D. Serrano-Velarde, "Future High Throughput Satellite systems," in IEEE First AESS European Conference on Satellite Telecommunications (ESTEL), Oct 2012.

[4] M. A. Vazquez, A. Perez-Neira, D. Christopoulos, S. Chatzinotas, B. Ottersten, P. Arapoglou, A. Ginesi, and G. Tarocco, "Precoding in Multibeam Satellite Communications: Present and Future Challenges," IEEE Wireless Communications, vol. 23, no. 6, pp. 88-95, December 2016.

[5] F. K. Nader Alagha and G. Montorsi, "Optimized non-orthogonal multiplexing in Peak Power Limited Channels," in 5th AIAA International Communications Satellite Systems Conference, International Communications Satellite Systems Conferences (ICSSC), Oct. 2017.

[6] X. Yan, H. Xiao, C. Wang, K. An, A. T. Chronopoulos, and G. Zheng, "Performance Analysis of NOMA-Based Land Mobile Satellite Networks," IEEE Access, vol. 6, pp. 31327-31339, 2018.

[7] H. Joudeh and B. Clerckx, "Robust Transmission in Downlink Multiuser MISO Systems: A Rate-Splitting Approach," IEEE Transactions on Signal Processing, vol. 64, no. 23, pp. 6227-6242, Dec 2016.

[8] M. A. Vázquez, M. Caus, and A. Pérez-Neira, "Rate Splitting for MIMO Multibeam Satellite Systems," in WSA 2018; 22nd International ITG Workshop on Smart Antennas, March 2018, pp. $1-6$.

[9] M. Caus, A. Pastore, M. Navarro, T. Ramirez, C. Mosquera, N. Noels, N. Alagha, and A. I. Perez-Neira, "Exploratory Analysis of Superposition Coding and Rate Splitting for Multibeam Satellite Systems," in 15th International Symposium on Wireless Communication Systems, Aug 2018.

[10] Y. Huang, C. Zhang, J. Wang, Y. Jing, L. Yang, and X. You, "Signal Processing for MIMO-NOMA: Present and Future Challenges," IEEE Wireless Communications, vol. 25, no. 2, pp. 32-38, April 2018.

[11] M. F. Hanif, Z. Ding, T. Ratnarajah, and G. K. Karagiannidis, "A Minorization-Maximization Method for Optimizing Sum Rate in the Downlink of Non-Orthogonal Multiple Access Systems," IEEE Transactions on Signal Processing, vol. 64, no. 1, pp. 76-88, Jan 2016.

[12] M. A. Vazquez, M. Caus, and A. Perez-Neira, "Performance Analysis of Joint Precoding and MUD Techniques in Multibeam Satellite Systems," in IEEE Global Communications Conference (GLOBECOM), Dec 2016.

[13] M. Caus, M. A. Vázquez, and A. Perez-Neira, "NOMA and interference limited satellite scenarios," in 50th Asilomar Conference on Signals, Systems and Computers, Nov 2016.

[14] W. Nam, D. Bai, J. Lee, and I. Kang, "Advanced interference management for 5G cellular networks," IEEE Communications Magazine, vol. 52, no. 5, pp. 52-60, May 2014.

[15] A. Guidotti and A. Vanelli-Coralli, "Geographical Scheduling for Multicast Precoding in Multi-Beam Satellite Systems," CoRR, vol. abs/1804.06614, 2018. [Online]. Available: http://arxiv.org/abs/1804.06614

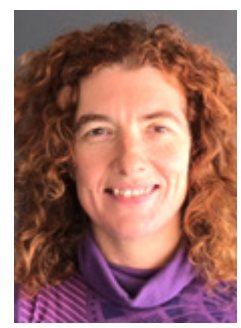

Ana I.Perez-Neira is full professor at Universitat Politècnica de Catalunya in the Signal Theory and Communication department since 2006 and was Vice rector for Research (2010-14). She is fellow researcher at Centre Tecnològic de Telecomunicacions de Catalunya, where she is the Coordinator of the SatCom and Space activities. Her research is in signal processing for communications, focused on satellite communications. She has more than 60 journal papers and 300 conference papers. She is coauthor of 7 books. She has leaded more than 20 projects and holds 8 patents. She is the coordinator of the Network of Excellence on satellite communications, financed by the European Space Agency: SatnexIV. She has been associate editor of the IEEE TSP and EURASIP SP and ASP. She is member of the BoG of the IEEE SPS. She is recipient for the 2018 EURASIP Society Award and she is the general chair of IEEE ICASSP'20.

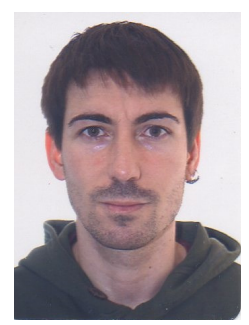

Marius Caus received the M.Sc. and PhD (cum laude) degrees in Telecommunications engineering from the Universitat Politècnica de Catalunya (UPC), Barcelona, Spain, in July 2008 and December 2013, respectively. In September 2009 he was granted by the Spanish Government to carry out his PhD studies in the Signal Theory and Communications Department at UPC. He has participated in several projects funded by the European Commission (PHYDYAS, EMPhAtiC, NEWCOM\# and FANTASTIC-5G) and the European Space Agency. He is currently a Researcher in Centre Tecnològic de Telecomunicacions de Catalunya (CTTC), Castelldefels, Spain. His main research interests include filter bank-based multicarrier systems, signal processing for communications and satellite communications.

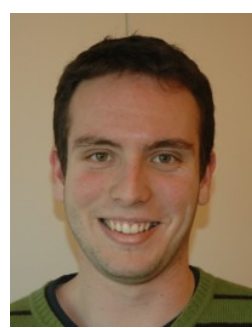

Miguel Angel Vazquez received the Telecommunication Engineering degree, the Master's degree, and the Ph.D. (cum laude) degree from Universitat Politècnica de Catalunya (UPC), Barcelona, Spain, in 2009, 2012, and 2014, respectively. He also holds a degree in Computer Science from National Distance Education University. In parallel to his Ph.D. studies, he worked as a research engineer at CTTC from January 2010. He was a visiting researcher at Patavina Technologies in the context of a Marie Curie Action. He has been key personnel in several projects funded by the European Commission, the European Space Agency, the Spanish Ministry of Science and industries. He has one patent owned by ESA. He is a member of the Networld2020 satellite communications experts group. He co-organized the 8th Advanced Satellite Multimedia Systems Conference and the 14th Signal Processing for Space Communications Workshop (ASMS-SPSC 2016) and IEEE Signal Processing School for Next Generation Satellite Systems (SPNGS 2016). 